\title{
Analysis of stress concentrations in multi-fibre microcomposites by means of Raman spectroscopy
}

\author{
P. W. J. VAN DEN HEUVEL $\dagger *$, T. PEIJS $\dagger$, R. J. YOUNG* \\ $\dagger$ Centre for Polymers and Composites, Eindhoven University of Technology, PO Box 513, 5600 MB Eindhoven, \\ The Netherlands \\ * Manchester Materials Science Centre, UMIST/University of Manchester, Grosvenor Street, Manchester M1 7HS, \\ $U K$
}

Many theories have been developed to predict the strength of unidirectional fibre-reinforced composites $[1-4]$. Over the years it has been recognized that knowledge of the stress concentrations in fibres adjacent to a broken fibre is of major importance in these theories. Since such stress concentrations cannot normally be measured directly, many analytical theories have been proposed for the calculation of this stress concentration factor [5-7]. Recently, also the finite element method has been used to calculate the stress concentration factor in fibres adjacent to a fibre break [8]. Because of the complexity of the problem, all these calculations contain great simplifications with respect to, for example, the fibre arrangement and material properties. Using a simple shear lag theory in which only the fibre is assumed to carry the tensile stress, Hedgepeth and Van Dyke [5] calculated a stress concentration factor of 1.333 for a planar array of fibres, independent of the inter-fibre spacing and the physical properties of the fibre and the matrix. More complicated theories for the stress concentration factor include, for example, the fibre content and the modulus of the fibre $\left(E_{\mathrm{f}}\right)$ and of the matrix $\left(E_{\mathrm{m}}\right)$ $[6,7]$. Ochiai et al. [6], for instance, proposed a theory for the stress concentration factor in a planar array of elastic fibres in an elastic matrix in which, in contrast to Hedgepeth and Van Dyke [5], both the fibres and the matrix are assumed to carry tensile stress and act as media for stress transfer. At an inter-fibre spacing of 1 fibre diameter $(\phi)$ this theory yields a stress concentration factor of approximately 1.25 for $E_{\mathrm{f}}=350 \mathrm{GPa}$ and $E_{\mathrm{m}}=3.5 \mathrm{GPa}$. Unfortunately, until now experimental verification of calculated stress concentration factors had not been possible.

In this letter, we present preliminary results showing that Raman spectroscopy makes it possible to measure stress concentration factors in fibres adjacent to a fibre break. With Raman spectroscopy, strain profiles of a large variety of fibres can be measured in situ, i.e. inside a composite [9-11]. In our experiments use is made of a carbon/epoxy multi-fibre microcomposite, in which five continuous carbon fibres are aligned parallel in a planar array at a predefined inter-fibre spacing in a dumbbell-shape tensile bar (see Fig. 1). Such a microcomposite has shown to be very useful in investigations into the effects of stress concentration factors due to an

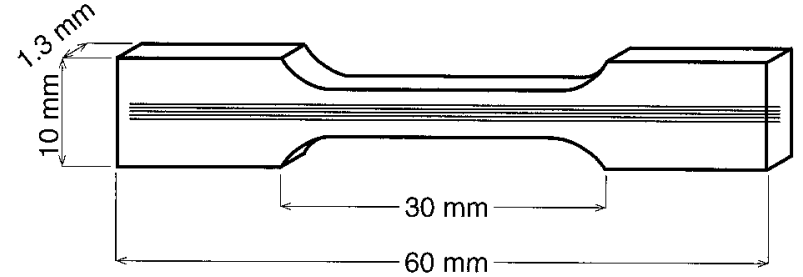

Figure I Schematic representation of the multi-fibre microcomposite containing a planar array of five carbon fibres in an epoxy tensile bar.

initial fibre break, i.e. fibre-fibre interactions, upon the failure process of fibre-reinforced composites [12-16].

The fibres used in this study were conventional PAN-based, surface-treated Tenax HMS-40 (Akzo Tenax Fibers GmbH \& Co. KG) carbon fibres $\left(E_{\mathrm{f}}=350 \mathrm{GPa}\right)$. The epoxy sizing of the fibres was removed by placing the fibres in acetone for several hours. The matrix consisted of a common diglycidyl ether of bisphenol A (DGEBA) type epoxy (Ciba Geigy, Araldite LY556) and a stoichiometric amount of poly(oxypropylene)tri-amine (Texaco, Jeffamine T-403) curing agent. In this letter preliminary results will be shown of only one of the microcomposites we have prepared so far. Preparation of the multifibre microcomposite is described in detail elsewhere [16]. The resin mixture was cured at room temperature for $24 \mathrm{~h}$ and post-cured at $75^{\circ} \mathrm{C}$ for $16 \mathrm{~h}$ $\left(E_{\mathrm{m}}=3.5 \mathrm{GPa}\right)$. Raman spectra were obtained from single carbon fibres both in air and in the microcomposite using a Raman spectrometer system (Renishaw 1000) coupled to an optical microscope (Olympus BH-2). The spectra were obtained using the $632.8 \mathrm{~nm}$ red line of a $25 \mathrm{~mW}$ helium-neon laser (Spectra Physics Model 127-25) focused to an approximately $2 \mu \mathrm{m}$ spot on the fibre surface. A Lorentzian fitting procedure was used to determine the positions of the strain sensitive $2660 \mathrm{~cm}^{-1}$ Raman bands. For deformation of the carbon fibres in air, single fibres were mounted on a straining rig using a cardboard frame, similar to the procedure described in ASTM D-3379. The gauge length of the fibres was approximately $100 \mathrm{~mm}$. The displacements of the fibres were determined to an accuracy of $\pm 5 \mu \mathrm{m}$ using a micrometer attached to the straining rig. For analysis of the multi-fibre microcomposite, the microcomposite was strained in a miniature tensile tester (Minimat, Polymer Labora- 
tory) equipped with a $1000 \mathrm{~N}$ load cell and using manual control. The strain on the microcomposite was monitored and controlled accurately using a strain gauge mounted directly on the specimen.

Fig. 2 shows the $2660 \mathrm{~cm}^{-1}$ Raman band for a fibre in air in the unstrained state and at a tensile strain of $0.75 \%$. As commonly observed the increase in tensile strain results in a clear shift of the peak to a lower wave number $[9-11]$. The dependence of the peak position of the $2660 \mathrm{~cm}^{-1}$ Raman band upon strain is shown in Fig. 3. This graph is used to convert the Raman spectra of the carbon fibres in the multi-fibre microcomposite into fibre strains.

In the multi-fibre microcomposite investigated in this letter the inter-fibre spacing varied from 0.8 3.1 $\phi$, i.e. $4-15 \mu \mathrm{m}$ (see Fig. 4). Fig. $4 \mathrm{a}$ shows the strain profiles of each of the five fibres at a matrix strain of $0 \%$. Measurements were taken at $50 \mu \mathrm{m}$ intervals over a length of $2600 \mu \mathrm{m}$ along the fibre. It is observed that the fibres are subjected initially to a compressive strain of approximately $0.1 \%$. This can probably be explained by the difference in thermal expansion coefficient between the fibre and the matrix which gives rise to thermal stresses after curing the sample [9]. In Fig. $4 \mathrm{~b}$ the macroscopic strain of the specimen has been increased to $0.9 \%$. Raman spectra were taken at $10 \mu \mathrm{m}$ intervals in the

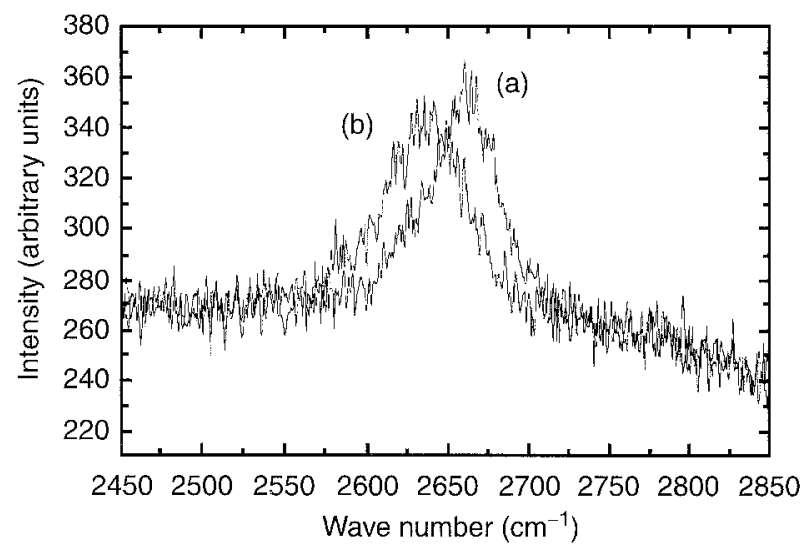

Figure 2 Raman spectra of a Tenax HMS-40 carbon fibre in air showing the $2660 \mathrm{~cm}^{-1}$ band at two levels of strain, $\epsilon$ : (a) $0 \%$; (b) $0.75 \%$.

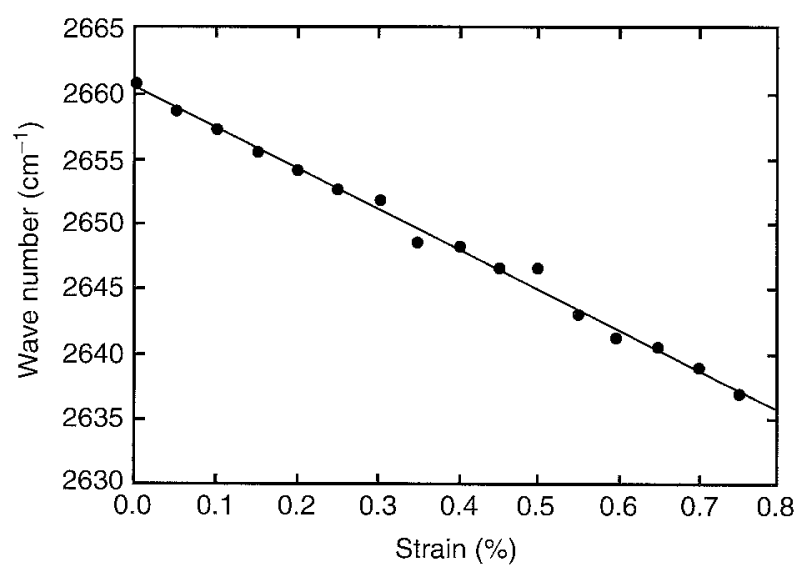

Figure 3 Dependence of the peak position of the $2660 \mathrm{~cm}^{-1}$ Raman band upon strain for Tenax HMS-40. vicinity of the break and at $20 \mu \mathrm{m}$ intervals away from the break. In the middle fibre (fibre 3) a break can be clearly observed. At both broken fibre ends the strain builds up to the plateau strain level over a distance of approximately $600 \mu \mathrm{m}$, i.e. the ineffective length.

More importantly, increased strains can be observed in the two fibres directly adjacent to the broken fibre over a distance of approximately $400 \mu \mathrm{m}$ along the fibre, i.e. the affected length. As expected the maximum strain is located at the level of the fibre break. From Fig. $4 \mathrm{~b}$ it is found that at an inter-fibre spacing of $0.8 \phi$ (fibre 4 ) the maximum strain concentration factor, $k$, is approximately 1.26 At a spacing of $2.0 \phi$ (fibre 2) the maximum strain concentration factor is approximately 1.20. At these inter-fibre spacings the theory proposed by Ochiai et al. [6] yields strain concentration factors of approximately 1.29 and 1.25 , respectively, for $E_{\mathrm{f}}=350 \mathrm{GPa}$ and $E_{\mathrm{m}}=3.5 \mathrm{GPa}$. Thus, there is good agreement between the theory proposed by Ochiai et al. [6] and the experimental results. With respect to the strain profiles of the two outer fibres (fibre 1 and fibre 5) no significant strain concentrations are observed. Apart from insufficient resolution, this might be due to the larger distance from the break $(3.7-3.9 \phi)$ or to shielding caused by the fibres in between the outer and the broken fibre (fibre 2 and fibre 4). Fig. 4c shows the strain profiles of each fibre at a matrix strain of $1.1 \%$ when further fragmentation has occurred. Alignment of breaks as a result of the increased strains around the initial fibre break is clearly observed for all fibres, as reported previously by Van den Heuvel et al. $[14,16]$ and Jones and DiBenedetto [15].

In conclusion, it has been shown that in a carbon epoxy multi-fibre microcomposite with a planar array of fibres direct measurement of strain concentration factors due to an initial fibre break is possible by means of Raman spectroscopy. It is believed that this result will be of great importance with respect to both a better understanding of failure in fibre-reinforced materials and to the development of theories describing the longitudinal strength of unidirectional composites in particular. Presently, further investigations into the influence of inter-fibre spacing and fibre surface-treatment upon the stress concentration factors are being performed and compared with the available theories as well as finite element calculations. We hope to report on this in the near future.

\section{Acknowledgements}

The authors would like to thank Huntsman N.V. Belgium for supplying the Jeffamine T-403 curing agent, Ciba Geigy (Duxford, UK) for supplying the Araldite LY556 resin and Akzo Fibers GmbH \& Co. $\mathrm{KG}$ for supplying the carbon fibres. One of the authors (R.J.Y.) is grateful to the Royal Society for support in the form of the Wolfson Research Professorship in Materials Science. This work was supported in part by a grant from the EPSRC. 

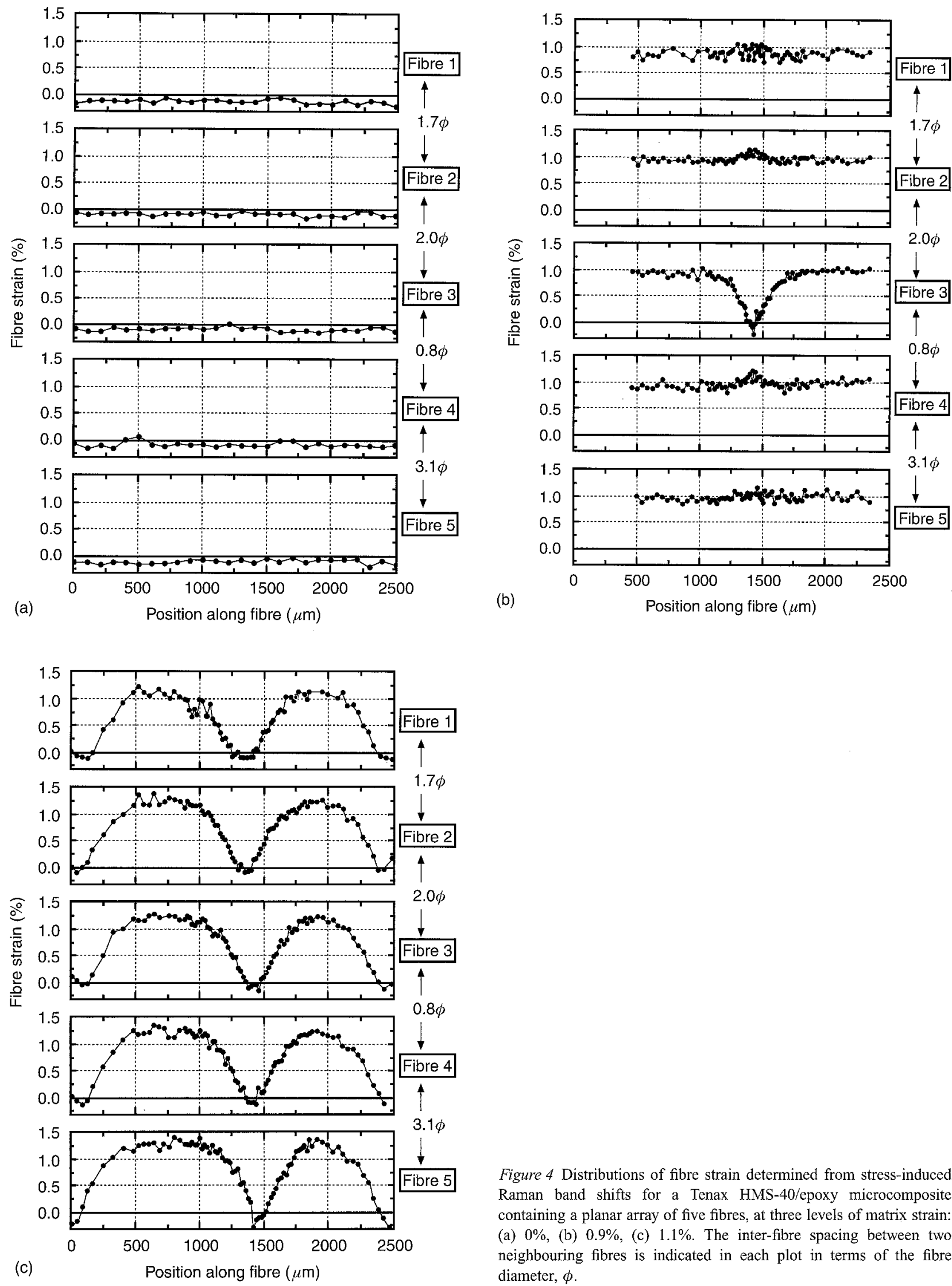

Figure 4 Distributions of fibre strain determined from stress-induced Raman band shifts for a Tenax HMS-40/epoxy microcomposite containing a planar array of five fibres, at three levels of matrix strain: (a) $0 \%$, (b) $0.9 \%$, (c) $1.1 \%$. The inter-fibre spacing between two neighbouring fibres is indicated in each plot in terms of the fibre diameter, $\phi$.

8. M. R. NEDELE and M. R. WISNOM, Composites 25 (1994) 549.

9. Y. HUANG and R. J. YOUNG, ibid. 26 (1995) 541.

10. M. C. ANDREWS, R. J. YOUNG and J. MAHY, Compos Interf. 2 (1994) 433

11. C. Vlattas and C. GAliotis, Polymer 35 (1994) 2335.

12. J. MULLin, J. M. BERRY and A. GATTI, J. Compos. Mater. 2 (1968) 82.

13. H. D. WAGNER and L. W. STEENBAKKERS, $J$. Mater 
Sci. 24 (1989) 3956.

14. P. W. J. VAN DEN HEUVEL, Y. J. W. VAN DER BRUGGEN and T. PEIJS, Adv. Compos. Lett. 3 (1994) 197.

15. K. D. Jones and A. T. Dibenedetto, Compos. Sci. Technol. 51 (1994) 53.
16. P. W. J. VAN DEN HEUVEL, Y. J. W. VAN DER BRUGGEN and T. PEIJS, Composites 27A (1996) 855.

Received 6 November 1995 and accepted 5 August 1996 